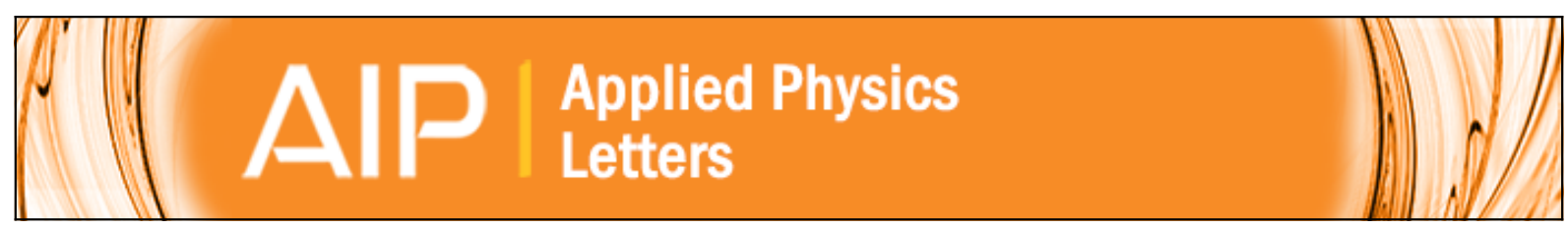

\title{
Microwave properties of nanodiamond particles
}

Daniel Slocombe, Adrian Porch, Etienne Bustarret, and Oliver A. Williams

Citation: Applied Physics Letters 102, 244102 (2013); doi: 10.1063/1.4809823

View online: http://dx.doi.org/10.1063/1.4809823

View Table of Contents: http://scitation.aip.org/content/aip/journal/apl/102/24?ver=pdfcov

Published by the AIP Publishing

$\stackrel{A}{A} \mathbb{P} P$ Re-register for Table of Content Alerts

Create a profile.

Sign up today! 


\title{
Microwave properties of nanodiamond particles
}

\author{
Daniel Slocombe, ${ }^{1,2}$ Adrian Porch, ${ }^{1}$ Etienne Bustarret, ${ }^{3}$ and Oliver A. Williams ${ }^{4}$ \\ ${ }^{1}$ School of Engineering, Cardiff University, Queen's Buildings, The Parade, Cardiff CF24 3AA, \\ United Kingdom \\ ${ }^{2}$ Inorganic Chemistry Laboratory, University of Oxford, South Parks Road, Oxford OXI 3QR, United Kingdom \\ ${ }^{3}$ Institut Néel, CNRS \& Université Joseph Fourier, 25 rue des Martyrs BP 166, 38042 Grenoble cedex 9. \\ France \\ ${ }^{4}$ School of Physics and Astrophysics, Cardiff University, Queen's Buildings, The Parade, Cardiff CF24 3AA, \\ United Kingdom
}

(Received 10 March 2013; accepted 23 May 2013; published online 17 June 2013)

\begin{abstract}
The dielectric properties of nanodiamond powders were characterised at microwave frequencies using a cavity perturbation technique, and results were compared with UV Raman spectroscopy. Surface $\mathrm{sp}^{2}$ hybridisation in the nanodiamond samples was varied by subsequent oxygenation and hydrogenation. Dielectric polarisation and loss increased as the $\mathrm{sp}^{2}$ hybridisation was increased. The sensitivity to surface bound $\mathrm{sp}^{2}$ carbon obtained by the microwave cavity technique far exceeds that of comparable techniques (such as Raman spectroscopy) and is much more convenient in practice, lending itself to studies of real-time modification of such powders by external influences (such as temperature and chemical functionalisation). (C) 2013 AIP Publishing LLC. [http://dx.doi.org/10.1063/1.4809823]
\end{abstract}

Nanodiamond particles have recently generated substantial interest in areas as diverse as diamond nucleation, biomarkers, lubrication, and drug delivery. ${ }^{1-4}$ The dielectric properties of nanodiamond particles have been sparsely studied due to the difficulty of contact electrode approaches as well as percolation issues when measuring nanoparticles. 5,6 Of particular interest is the degree of $\mathrm{sp}^{2}$ hybridisation of the resulting $\mathrm{C}-\mathrm{C}$ bonds versus the $\mathrm{sp}^{3}$ hybridisation expected in the interior of bulk diamond. In this work, a microwave cavity perturbation technique is proposed to measure the effective relative permittivity of treated nanodiamond powders, which does not require direct electrical contacts to be made to the particles.

Commercial detonation nanodiamond powders were sourced from PlasmaChem $\mathrm{GmbH}$ (G01 grade). This material is purified from the detonation product by the supplier with multiple acid treatments. The core particle size is approximately $5 \mathrm{~nm}$. Three nanodiamond samples were made from this material. First, untreated powders were measured, designated $U$ in what follows. Samples were then oxidised by burning the powders in air (designated $\mathrm{O}$ ), as has been demonstrated by Osswald et $a l^{7}$ and Hees et al. ${ }^{8}$ Finally, samples were hydrogenated (designated $\mathrm{H}$ ) by annealing in hydrogen gas at $10 \mathrm{mbar}, 400^{\circ} \mathrm{C} .{ }^{9}$ Both $\mathrm{O}$ and $\mathrm{H}$ samples were measured in the microwave cavity. As a comparison, the same volume of carbon black powder (sourced from Alfa Aesar and designated as CB) was also measured.

Micro-Raman spectra were measured in air with a liquid nitrogen cooled UV enhanced CCD detector in the backscattering parallel geometry. A small amount of diamond powder was placed under a $\times 40 \mathrm{UV}$ objective using the $325 \mathrm{~nm}$ line of a $\mathrm{HeCd} \mathrm{CW}$ laser. The incident power was reduced to the point where there was no drift in the spectral signatures over various acquisition times (typ. 1-20 min). This clearly limited the signal to noise ratio below the value where surface species such as $\mathrm{CH}_{x}$ could be detected at higher Stokes shifts. ${ }^{10}$ The spectra of all of the particles as seen in Fig. 1 were very similar, exhibiting clear peaks at $1332 \mathrm{~cm}^{-1}$, characteristic of $\mathrm{sp}^{3}$ bonding. This peak is broadened by the small size of the crystallites as well as convoluted with the D-band. A strong G-band is evident at higher wavenumbers due to $\mathrm{sp}^{2}$ bonding, as is a shoulder below the $1332 \mathrm{~cm}^{-1}$ peak due to transpolyacetylene at the grain surfaces. ${ }^{11}$ It is clear that there is progressively lower intensity of these $\mathrm{sp}^{2}$ signatures (shoulder below $1332 \mathrm{~cm}^{-1}$ and G-band) between the untreated, hydrogen annealed, and air annealed samples respectively. This difference is, however, subtle due to the insensitivity of UV Raman spectroscopy to small surface concentrations of $\mathrm{sp}^{2} .{ }^{10}$ Attempts at using larger wavelengths (up to $633 \mathrm{~nm}$ ) in Raman spectroscopy were unable to distinguish between the different samples.

All microwave measurements are performed in a cylindrical host cavity, shown schematically in Fig. 2. Powdered samples are placed in thin-walled quartz tubes and are

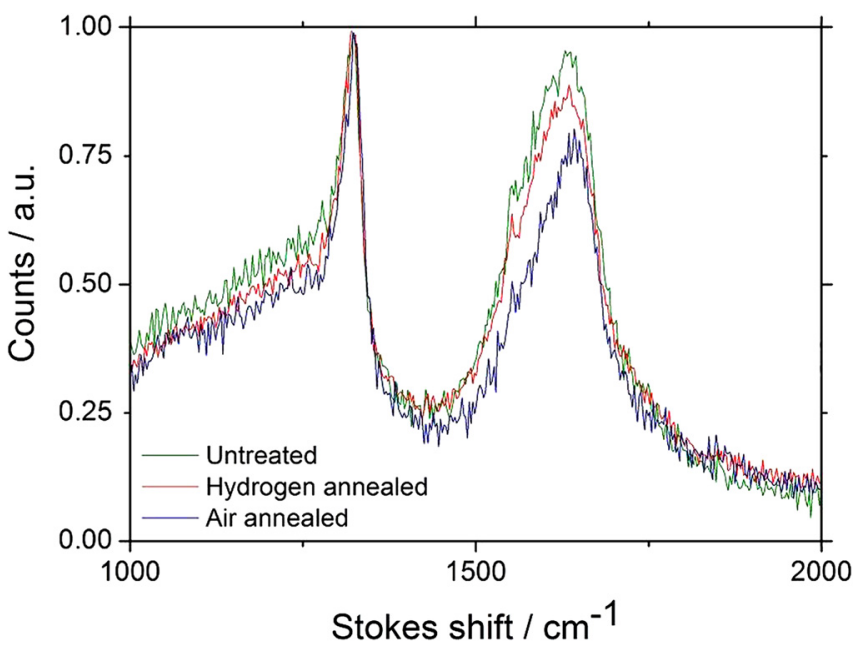

FIG. 1. Raman spectra of the three different diamond particle types. 
(a) $\mathrm{TM}_{010}$ mode

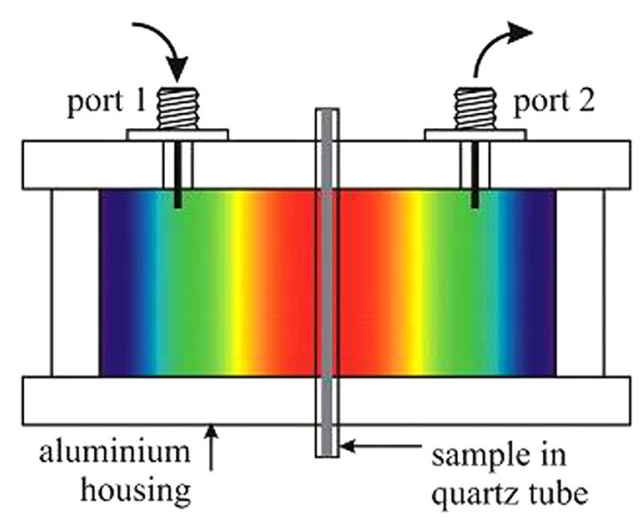

(b) $\mathrm{TM}_{020}$ mode

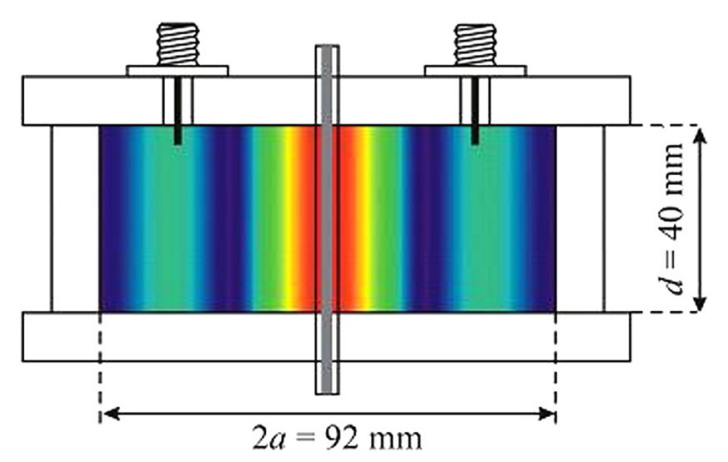

FIG. 2. Schematic diagram of the aluminium host cavity, also showing the magnitude of the electric field in (a) the $\mathrm{TM}_{010}$ mode, and (b) the $\mathrm{TM}_{020}$ mode. In both cases, the field is parallel to the axis of the cylinder. Samples are introduced along the axis, contained in a thin-walled quartz capillary of inner diameter $2 \mathrm{~mm}$. For such small capillaries, we may assume that the applied field is uniform for both modes.

introduced along the axis of the cavity via small holes drilled in the top and bottom plates. The cavity is machined in three parts from bulk aluminium (for high quality factor $Q$ ), with top and bottom plates attached firmly to the cylindrical section using M10 bolts, thus, ensuring good electrical connection at the joints thus formed. The internal dimensions of the cavity are radius $a=4.6 \mathrm{~cm}$ and length $d=4.0 \mathrm{~cm}$, as indicated in Fig. 2.

The $\mathrm{TM}_{010}$ and $\mathrm{TM}_{020}$ modes are chosen for dielectric property measurement of the samples owing to the high, uniform electric field near the cavity axis. ${ }^{12}$ The electric field in these modes is directed parallel to the axis, thus, parallel to the axis of the sample tube, resulting in only minor modifications of the local electric field in the presence of the quartz tube. The distribution of electric field magnitude for each mode is also shown in Fig. 2. For tubes of inner radii $r \ll a$, we may assume the applied electric field to be uniform for each mode. Here, $r / a=0.022$ so the uniform field approximation is a good one. The radius $a$ is chosen so that the $\mathrm{TM}_{010}$ mode has a resonant frequency of about $2.5 \mathrm{GHz}$. The cavity aspect ratio $d / a$ is chosen to be small enough for the $\mathrm{TM}_{010}$ mode to be the dominant mode (i.e., for it to be well below the resonant frequency of the $\mathrm{TE}_{111}$ mode), but not too small to significantly compromise the high $Q$ factor or the axial uniformity of the electric field.
Cavity excitation is provided by a pair of square-flanged SMA jack connectors (supplied by Farnell Ltd.), at radial positions $3 \mathrm{~cm}$ from the axis. These have open circuit terminations, with an extended center conductor providing capacitive coupling to the electric field of the TM cavity modes. All microwave measurements are performed using an Agilent E5071B network analyser, controlled using LABVIEW software (National Instruments Ltd.). Measurements are performed of the transmitted microwave power $\left|S_{21}\right|^{2}$ in the frequency domain, and non-linear, least-squares curve fitting to a Lorentzian response is used to determine resonant frequencies and loaded quality factors (denoted as $Q_{\mathrm{L}}$ ). We remove the effects of cavity couplings by converting $Q_{\mathrm{L}}$ into the unloaded quality factor $Q$ in each case. The cavity couplings are adjusted so that they are equal (i.e., symmetric) and so the conversion is made using the simple formula $Q=Q_{\mathrm{L}}(1-$ $10^{-I L / 20}$ ), where $I L$ is the insertion loss (i.e., transmitted power) at resonance, measured in $\mathrm{dB} .{ }^{13}$ Furthermore, the couplings are kept weak in all circumstances (with $I L<-30$ $\mathrm{dB}$ ), meaning that very little systematic error is introduced as a result of this decoupling process.

The theoretical values of the resonant frequencies of the $\mathrm{TM}_{010}$ and $\mathrm{TM}_{020}$ modes of the empty cavity are calculated using $f_{0 n 0}=c p_{0 n} / 2 \pi a$, where $p_{01}=2.405$ and $p_{02}=5.520$ are the first two zeros of the Bessel function $J_{0}(x)$, giving values of 2.495 and $5.726 \mathrm{GHz}$, respectively. The measured values are found to be 2.501 and $5.730 \mathrm{GHz}$, respectively, in excellent agreement with these theoretical values. The unloaded $Q$ factors are measured to be 10140 and 14890 for the $\mathrm{TM}_{010}$ and $\mathrm{TM}_{020}$ modes, respectively, at an ambient temperature of $25^{\circ} \mathrm{C}$. Assuming a resistivity for aluminium of $2.8 \mu \Omega \mathrm{cm}$, the corresponding theoretical values of the unloaded $Q$ factors are 12700 and 19 200, respectively. The measured $Q$ factors are lower than the theoretical values by about $25 \%-30 \%$, as is typical in metal cavities made by traditional machining methods. This is due to the combined effects of increased surface area due to surface roughness (since the skin depth in $\mathrm{Al}$ at 2.50 and $5.73 \mathrm{GHz}$ is only 1.7 and $1.1 \mu \mathrm{m}$, respectively), increased resistivity of the surface layer due to a high dislocation density and surface discontinuities due to cavity construction.

The quartz sample tubes were supplied by VirtoCom Ltd., and have inner and outer diameters of 2.0 and $2.4 \mathrm{~mm}$, respectively. An empty tube was found to reduce the resonant frequencies of the $\mathrm{TM}_{010}$ and $\mathrm{TM}_{020}$ modes by 2.2 and $11.9 \mathrm{MHz}$, respectively, with negligible reduction in $Q$ factors. This makes them excellent for sensitive measurements of the dielectric properties of powders using the cavity perturbation technique. We have measured the complex permittivity of quartz at $25^{\circ} \mathrm{C}$ using these tubes in the same cavity, giving $\varepsilon=3.80-j 0.0003$ at $2.5 \mathrm{GHz}$, i.e., a loss tangent of less than $10^{-4}$.

The sample tubes were filled to a depth of $5 \mathrm{~cm}$ with the powder samples $(4 \mathrm{~cm}$ of which will be in the active region of the cavity), and all samples were left to settle overnight before being measured. The active sample volume is therefore $0.126 \mathrm{~cm}^{3}$. The measured resonant traces showing $\left|S_{21}\right|^{2}$ as a function of frequency are shown in Fig. 3. Only data for the $\mathrm{TM}_{010}$ mode are shown here, but the $\mathrm{TM}_{020}$ mode exhibits the same qualitative behaviour and data are recorded for both modes. 

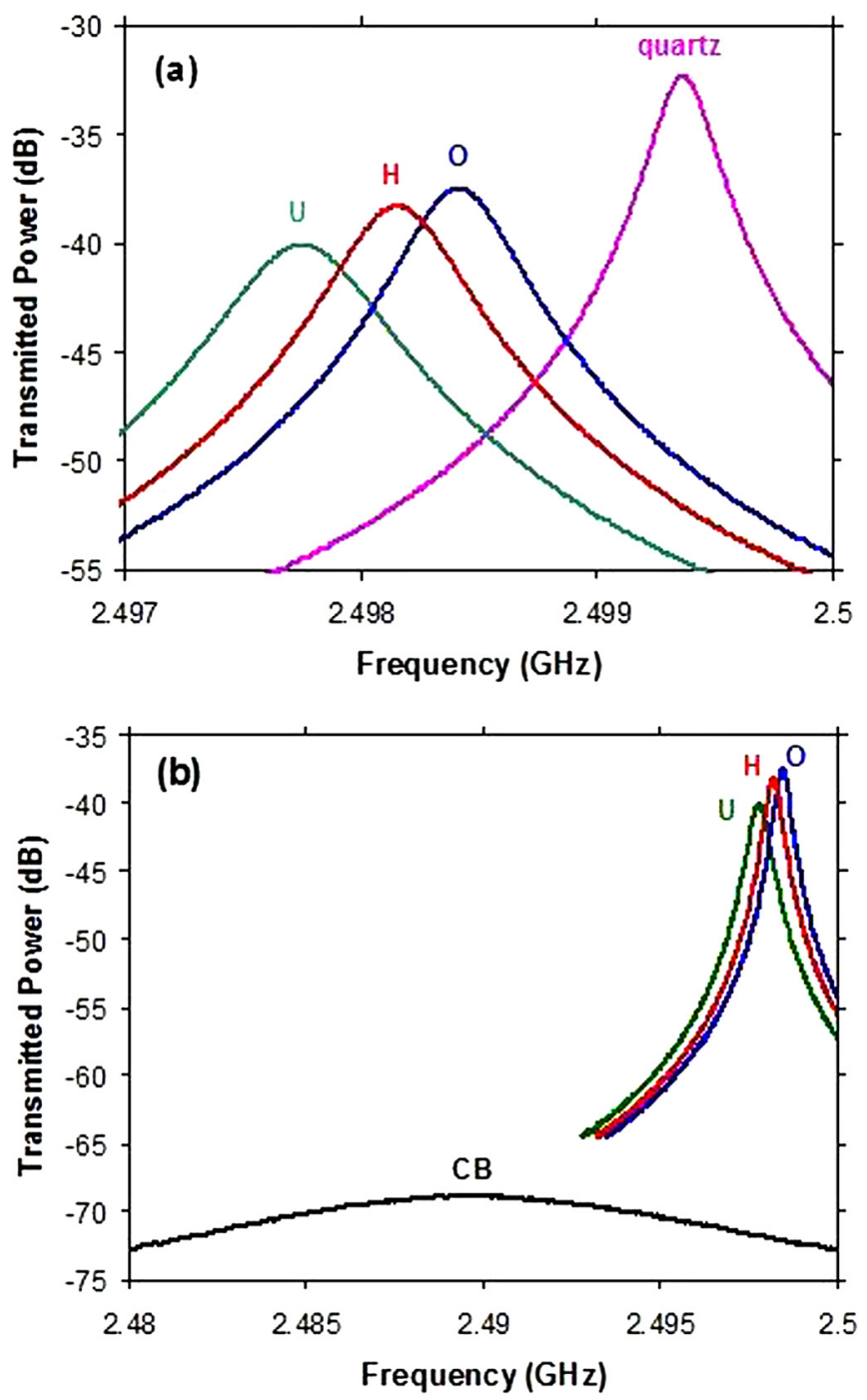

FIG. 3. Resonant traces showing the transmitted power $\left|\mathrm{S}_{21}\right|^{2}$ as a function of frequency for the $\mathrm{TM}_{010}$ mode. Power samples are contained in thinwalled quartz tubes and the changes in resonant frequency and $3 \mathrm{~dB}$ bandwidths are measured relative to the empty tubes. (a) The response of an empty quartz tube, together with quartz tubes filled with three nanodiamond samples: one untreated $(\mathrm{U})$, one oxygenated $(\mathrm{O})$, and another hydrogenated (H). (b) The responses of the same three nanodiamond samples, this time compared with the same volume of $\mathrm{CB}$.

To analyse this data, we first define the complex relative permittivity of a dielectric material as $\varepsilon=\varepsilon_{1}-j \varepsilon_{2}$, where $\varepsilon_{1}$ (or, more properly, $\varepsilon_{1}-1$ ) quantifies the polarisation of the dielectric material in an applied electric field, whilst $\varepsilon_{2}$ quantifies the dielectric loss. We use first order cavity perturbation theory to relate the changes in the resonator parameters to the dielectric properties of a sample inserted into the electric field antinode of the cavity. ${ }^{13}$ We first define $f_{0}$ as the resonant frequency (for either mode) and $f_{\mathrm{B}}=f_{0} / Q$ as the unloaded $3 \mathrm{~dB}$ (or half-power) bandwidth. If a sample is found to reduce the resonant frequency by an amount $\Delta f_{0}$ and increase the $3 \mathrm{~dB}$ bandwidth by $\Delta f_{\mathrm{B}}$, these are related to the complex permittivity of the sample by the approximate formulae

$$
\begin{gathered}
\frac{\Delta f_{0}}{f_{0}} \approx\left(\varepsilon_{1}-1\right) \frac{V_{\mathrm{s}}}{2 V_{\mathrm{eff}}}, \\
\frac{\Delta f_{B}}{f_{0}}=\Delta\left(\frac{1}{Q}\right) \approx \varepsilon_{2} \frac{V_{\mathrm{s}}}{V_{\mathrm{eff}}},
\end{gathered}
$$

where $f_{0}$ is the unperturbed resonant frequency, $V_{\mathrm{s}}$ is the sample volume, and $V_{\text {eff }}$ is the effective volume of the electric field energy within the cavity. The latter quantity is defined by the volume integral

$$
V_{\text {eff }}=\int_{V} \frac{E^{2}}{E_{0}^{2}} d V,
$$

where $E_{0}$ is the electric field at the position of the sample. Evaluating Eq. (3) from the theoretical electric field distributions gives $V_{\text {eff }}=\pi a^{2} L J_{1}^{2}\left(p_{0 n}\right)$ for the $\mathrm{TM}_{0 n 0}$ modes, i.e., effective volumes of $71.7 \mathrm{~cm}^{3}$ and $30.8 \mathrm{~cm}^{3}$ for the $\mathrm{TM}_{010}$ and $\mathrm{TM}_{020}$ modes, respectively.

Alternatively, the effective volume can be determined experimentally by the calibration measurement of a known material of known geometry. We use stainless steel spheres, since a metal sphere will develop a known electric dipole moment $p=4 \pi \varepsilon_{0} b^{3} E_{0}$ (where $b$ is the sphere radius) and then Eq. (1) reduces to $\Delta f_{0} / f_{0} \approx 2 \pi b^{3} / V_{\text {eff }}$. We measure the frequency shifts of seven sizes of individual spheres with radii $b$ in equal steps from $0.5 \mathrm{~mm}$ to $3.5 \mathrm{~mm}$, each positioned centrally in the cavity, then we use linear regression to evaluate $V_{\text {eff }}$ for each mode. This experimental process yields $V_{\text {eff }}=68.3 \pm 0.7 \mathrm{~cm}^{3}$ and $28.9 \pm 0.3 \mathrm{~cm}^{3}$ for the $\mathrm{TM}_{010}$ and $\mathrm{TM}_{020}$ modes, respectively. These are a few $\%$ smaller than the calculated values owing to the slight reduction of the electric field in the vicinity of the axial sample holes, and the small distortion of field around the coupling probes (since introducing any non-uniformity in the electric field results in a reduction of $V_{\text {eff }}$, as can be seen directly from Eq. (3)). We use these experimental values for $V_{\text {eff }}$ in all of our calculations based on Eqs. (1) and (2).

The experimental cavity perturbations $\Delta f_{0}$ and $\Delta f_{\mathrm{B}}$ and the calculated values of complex permittivity for the three nanodiamond samples $(\mathrm{U}, \mathrm{O}$, and $\mathrm{H}$ ) sample are shown in Table I (for the $\mathrm{TM}_{010}$ mode at $2.5 \mathrm{GHz}$ ) and Table II (for the $\mathrm{TM}_{020}$ mode at $5.73 \mathrm{GHz}$ ), together with the $\mathrm{CB}$ powder for comparison. These perturbations are measured relative to the same empty quartz tube in each case. We find that the volumes of the tubes vary by approximately $\pm 1 \%$, which accounts for the largest systematic error in the calculation of permittivity. Here, we quote values for the effective permittivity $\varepsilon_{\text {eff }}=\varepsilon_{1, \text { eff }}-j \varepsilon_{2, \text { eff }}$ of the powders, since most of the space within the sample tube is air and an effective medium approach is needed to extract the intrinsic permittivity $\varepsilon$ of

TABLE I. Change in resonant frequency and $3 \mathrm{~dB}$ bandwidth (relative to an empty quartz tube) on introducing the each sample into the $\mathrm{TM}_{010}$ mode at $2.5 \mathrm{GHz}$. Also shown are the resulting (effective) values of the complex permittivity. The random errors in these two experimental parameters (assessed by repeated measurement) are small, around $\pm 1 \%$. Variation in the volumes of the quartz tubes introduces an additional systematic error of approximately $\pm 1 \%$, resulting in an overall error of approximately $\pm 1.4 \%$ in the calculated values of permittivity.

\begin{tabular}{lcccc}
\hline \hline Sample & $\Delta f_{0}(\mathrm{MHz})$ & $\Delta f_{\mathrm{B}}(\mathrm{MHz})$ & $\varepsilon_{1, \mathrm{eff}}-1$ & $\varepsilon_{2, \mathrm{eff}}$ \\
\hline Untreated $(\mathrm{U})$ & $1.73 \pm 0.02$ & $0.353 \pm 0.004$ & $0.75 \pm 0.01$ & $0.077 \pm 0.002$ \\
Oxygenated $(\mathrm{O})$ & $1.08 \pm 0.01$ & $0.204 \pm 0.002$ & $0.47 \pm 0.01$ & $0.044 \pm 0.001$ \\
Hydrogenated $(\mathrm{H})$ & $1.32 \pm 0.01$ & $0.246 \pm 0.002$ & $0.57 \pm 0.01$ & $0.053 \pm 0.001$ \\
$\mathrm{CB}$ & $9.8 \pm 0.1$ & $17.0 \pm 0.2$ & $4.27 \pm 0.06$ & $3.69 \pm 0.05$ \\
\hline
\end{tabular}


TABLE II. Change in resonant frequency and $3 \mathrm{~dB}$ bandwidth (relative to an empty quartz tube) on introducing the sample into the $\mathrm{TM}_{020}$ mode at $5.73 \mathrm{GHz}$, with resulting (effective) values of complex permittivity. Errors are the same $\%$ values as for Table I.

\begin{tabular}{lcccc}
\hline \hline Sample & $\Delta f_{0}(\mathrm{MHz})$ & $\Delta f_{\mathrm{B}}(\mathrm{MHz})$ & $\varepsilon_{1, \text { eff }}-1$ & $\varepsilon_{2, \text { eff }}$ \\
\hline Untreated (U) & $8.88 \pm 0.09$ & $2.19 \pm 0.02$ & $0.71 \pm 0.01$ & $0.088 \pm 0.002$ \\
Oxygenated (O) & $5.55 \pm 0.06$ & $1.00 \pm 0.01$ & $0.45 \pm 0.01$ & $0.040 \pm 0.001$ \\
Hydrogenated (H) & $6.78 \pm 0.07$ & $1.30 \pm 0.01$ & $0.55 \pm 0.01$ & $0.052 \pm 0.001$ \\
CB & $70.3 \pm 0.7$ & $103 \pm 1$ & $5.64 \pm 0.07$ & $4.15 \pm 0.06$ \\
\hline \hline
\end{tabular}

the actual powder grains. The untreated sample $(\mathrm{U})$ has the largest polarisation $\varepsilon_{1, \text { eff }}-1$ and loss $\varepsilon_{2, \text { eff }}$ of the three nanodiamond samples. Oxygenation of the original sample (O) causes these to reduce, and subsequent hydrogenation $(\mathrm{H})$ leads to a partial return to the untreated state.

As can be seen from the results for the CB sample, a high degree of $\mathrm{sp}^{2}$ hybridisation causes large values of both microwave polarisation $\varepsilon_{1, \text { eff }}-1$ and loss $\varepsilon_{2, \text { eff }}$. Using this as a benchmark, we conclude that the greatest degree of $\mathrm{sp}^{2}$ hybridisation is found in the untreated sample (U), as we might have expected. Annealing in oxygen $(\mathrm{O})$ removes the most $\mathrm{sp}^{2}$ bonding, followed by hydrogen annealing $(\mathrm{H})$.

In order to quantify our analysis, and so relate the microwave results to the physical structure of the nanoparticles, we have developed a simple, quasistatic model. In this, we assume that each nanoparticle is spherical, with a single crystal diamond core (i.e., which is fully $\mathrm{sp}^{3}$ hybridised), each with an outer shell which is fully $\mathrm{sp}^{2}$ hybridised. We then apply the Maxwell-Garnett effective medium model ${ }^{14}$ to develop the effective permittivity $\varepsilon_{\text {eff }}$ of a macroscopic powder composed of close-packed, layered nanoparticles. This is the effective permittivity that has been measured and is presented in Tables I and II.

For the relative permittivity of the $\mathrm{sp}^{2}$ shell layer in the range $\left|\varepsilon_{\mathrm{s}}\right|<100$, our model shows that the values of $\varepsilon_{2, \text { eff }}$ in Tables I and II are directly proportional (to a systematic error of less than $5 \%$ ) to the volume fractions of $\mathrm{sp}^{2}$ hybridised material as one would expect. Here, we assume that the diamond core has a negligible microwave loss in comparison with the outer shell layer. Hence, from the combined results of Tables I and II, we deduce that the O sample has the smallest volume of $\mathrm{sp}^{2}$ hybridised carbon, the $\mathrm{H}$ sample has $(53 / 42)-1=26 \%$ more, whilst the U sample has $(83 / 42)-1$ $=98 \%$ more. Such values can be deduced directly from the changes in resonant bandwidth and so are subject to experimental errors of less than $\pm 3 \%$, which emphasises the power and convenience of this microwave absorption technique for such nanodiamond characterisation. Absolute values of the volume fractions are subject to large uncertainties owing to the experimental uncertainty in extracting representative values of $\varepsilon_{\mathrm{S}}$ from the carbon black data. Also, layer thicknesses may not be meaningful, since the $\mathrm{sp}^{2}$ layer itself may not be continuous. However, the ability to study such small variations in the volume of surface bound $\mathrm{sp}^{2}$ carbon present is of great benefit.

We have a high degree of confidence in using these microwave measurements to characterise the degree of $\mathrm{sp}^{2}$ hybridisation in nanodiamond samples and find that the trends are highly reproducible. There is only a slight frequency dependence in the properties between $2.5 \mathrm{GHz}$ and $5.7 \mathrm{GHz}$, so in practice either cavity modes can be used for these measurements.

Very few other techniques are able to study such small variations in surface bound $\mathrm{sp}^{2}$ with a comparable degree of sensitivity and simplicity. The Raman spectra of Fig. 1 demonstrate the difficulty in measuring such small changes and are also vulnerable to heating effects and damage of the particles by the laser irradiation (particularly visible Raman). This approach is highly reproducible, simple, and can be quantitative with further investigations and model development.

O.A.W. acknowledges Marie Curie actions for his IntraEuropean Fellowship "DIAMEMS". This work was also supported by EPSRC grants EP/J009814/1 and EP/K007459/1 as well as Royal Society Research Grant RG110577.

${ }^{1}$ O. A. Williams, O. Douheret, M. Daenen, K. Haenen, E. Osawa, and M. Takahashi, Chem. Phys. Lett. 445(4-6), 255-258 (2007).

${ }^{2}$ J. I. Chao, E. Perevedentseva, P. H. Chung, K. K. Liu, C. Y. Cheng, C. C. Chang, and C. L. Cheng, Biophys. J. 93(6), 2199-2208 (2007).

${ }^{3}$ R. Lam and D. Ho, Expert Opin. Drug Delivery 6(9), 883-895 (2009).

${ }^{4}$ V. N. Mochalin, O. Shenderova, D. Ho, and Y. Gogotsi, Nat. Nanotechnol. 7(1), 11-23 (2012).

${ }^{5}$ A. Chaudhary, J. O. Welch, and R. B. Jackman, Appl. Phys. Lett. 96(24), 242903 (2010).

${ }^{6}$ S. A. Maksimenko, V. N. Rodionova, G. Y. Slepyan, V. A. Karpovich, O. Shenderova, J. Walsh, V. L. Kuznetsov, I. N. Mazov, S. I. Moseenkov, A. V. Okotrub, and P. Lambin, Diamond Relat. Mater. 16(4-7), 1231-1235 (2007).

${ }^{7}$ S. Osswald, G. Yushin, V. Mochalin, S. O. Kucheyev, and Y. Gogotsi, J. Am. Chem. Soc. 128(35), 11635-11642 (2006).

${ }^{8}$ J. Hees, A. Kriele, and O. A. Williams, Chem. Phys. Lett. 509, 12 (2011).

${ }^{9}$ O. A. Williams, J. Hees, C. Dieker, W. Jager, L. Kirste, and C. E. Nebel, ACS Nano 4(8), 4824-4830 (2010).

${ }^{10}$ S. Ghodbane, A. Deneuville, D. Tromson, P. Bergonzo, E. Bustarret, and D. Ballutaud, Phys. Status Solidi A 203(10), 2397-2402 (2006).

${ }^{11}$ A. C. Ferrari and J. Robertson, Phys. Rev. B 63(12), 121405 (2001).

${ }^{12}$ A. C. Metaxas and R. J. Meredith, Industrial Microwave Heating (P. Peregrinus on behalf of the Institution of Electrical Engineers, London, UK, 1983).

${ }^{13}$ O. Klein, S. Donovan, M. Dressel, and G. Gruner, Int. J. Infrared Millim. Waves 14(12), 2423-2457 (1993).

${ }^{14}$ T. C. Choy, Effective Medium Theory: Principles and Applications, International Series of Monographs on Physics (Oxford University Press, Oxford, UK, 1999). 